\title{
Elementary Connection Mode for Distribution Network
}

\author{
Jiacheng $\mathrm{Li}$ \\ North China Electric Power University, Beijing, 102206 \\ alanncepu@foxmail.com
}

Keywords: Distribution network, Elementary connection

\begin{abstract}
By analysis of traditional connection mode and assessment system for distribution network, a new connection mode by use of the principle of elementary connection for distribution network is presented in this paper. A feasible connection mode of distribution network is constructed by use of various combination of elementary connection variables and inherent constraint conditions in regions of electricity consumption.
\end{abstract}

\section{Introduction}

With the rapid development of economy, the demand for electricity is growing, and requirements of reliability and economy performances in Electricity are more stringent. How to choose the optimal connection mode is an important piece of work in the planning and construction of MV power distribution network structure. The structure and connection of distribution network is complicated, and traditional mode only analyses the reliability and economy of it. The selection of connection is based on planners' personal experience and subjective judgment, which is unconvincing.

Some scholars have made studies in this field. For example reference [1] classifies connection modes of MV power distribution network according to Structured Element Theory, which provides new ideas to MV power distribution network planning. Reference [2] constructs a feasible connection mode of distribution network by various combination of elementary connections, proposes a dynamic evaluation model for elementary connection and achieves typical connection modes for distribution network under different boundary conditions.

This paper proposes a new method of constructing distribution network. Elementary connection mode for distribution network offers a new thought and detailed steps.

\section{Elementary Connection Mode for Distribution Network}

Elementary Connection Method. Mathematical thinking is the deep understanding of the nature of mathematical science and law, including the classification discussed ideas, equivalent transformation of ideas, thoughts, etc., these ideas are the basis of the need to trace the fundamental principle of knowledge, this is the embodiment of primitive thinking. "Yuan" refers to some kind of independent existence, "primitive" refers to the basic independent existence. Primitive is, the elements of a whole is the basis of understanding the overall, and primitive thinking is the basic principle of mining knowledge, grasp the essence of knowledge. It often has a general and a wide range of mobility and profound essential.

The definition of different areas of primitives is different. In physics, the earliest primitive refers to constitute the basic unit of substances, including electronic, quarks and neutrinos; In biology, will form the local area on the biological macromolecular structure characteristic sequence, to adapt to the combination between macromolecules (or fit), the basic structure unit, known as primitives, including zinc finger, leucine zipper, spiral - corner - screw and screw - ring - screw, etc., these structures is one of the important research topic in life science. The primitive theory has been widely in many areas, such as: using the primitive features of the concept of automatic text classification, image segmentation by texture primitives, using the combination of the primitive shape reconstruction and so on. A primitive theory as the research of the essence of things thought gradually be applied to more and more fields. 
Element is defined as the basic unit of energy transportation in distribution network, which means the simplest power supply mode in connection mode. For the aspect of structure, element usually consists of power source, load, line or several elements. The structure of elementary is shown in Fig. 1.

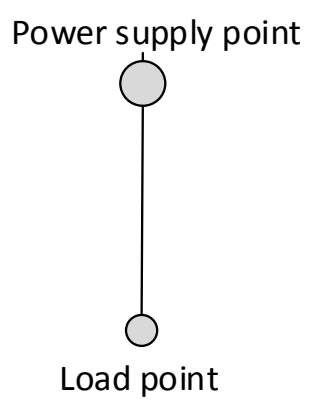

Figure 1. Structure of element

Elementary connection is the most basic and indivisible part of distribution network, which shows its characteristics as a whole to the outside. Several elements can be combined to form complex and diverse distribution network according to certain principles, as is shown in Fig. 2.
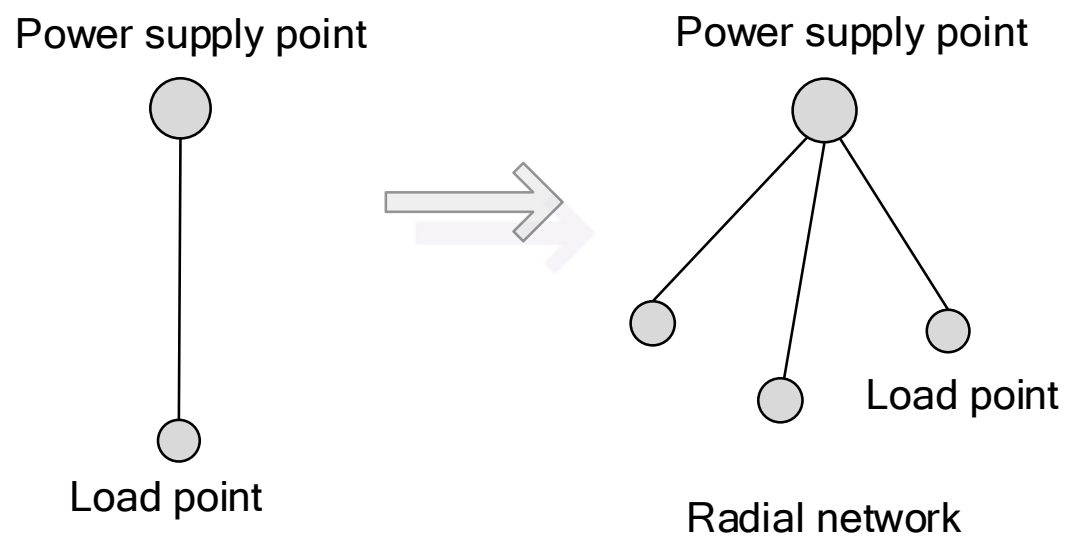

Figure 2. Diverse distribution network by combination of elements

Combination of Elements. Any complex network $U$ in planned area has equivalent elementary connection mode correspondingly. Each kind of connection and its parameters constitutes an elementary collection (elementary variables), while each elementary variable contains all categories of parameters of this kind of connection.

In elementary connection combination, elementary combined variable $\mathrm{T}(\mathrm{x})$ is characterized by a 4-element array (x, $U, G, M)$, as is shown in Fig. 3. Among them, $x_{i}(i=1,2 \ldots n)$ means different category of elementary connection, $U_{i}(i=1,2 \ldots n)$ means the objective factors of the planned regions where elementary connections combine, $G_{i}(i=1,2 \ldots n)$ means different load property of one certain planned district, $\mathrm{M}_{\mathrm{i}}(\mathrm{i}=1,2 \ldots \mathrm{n})$ means corresponding constraint conditions. 


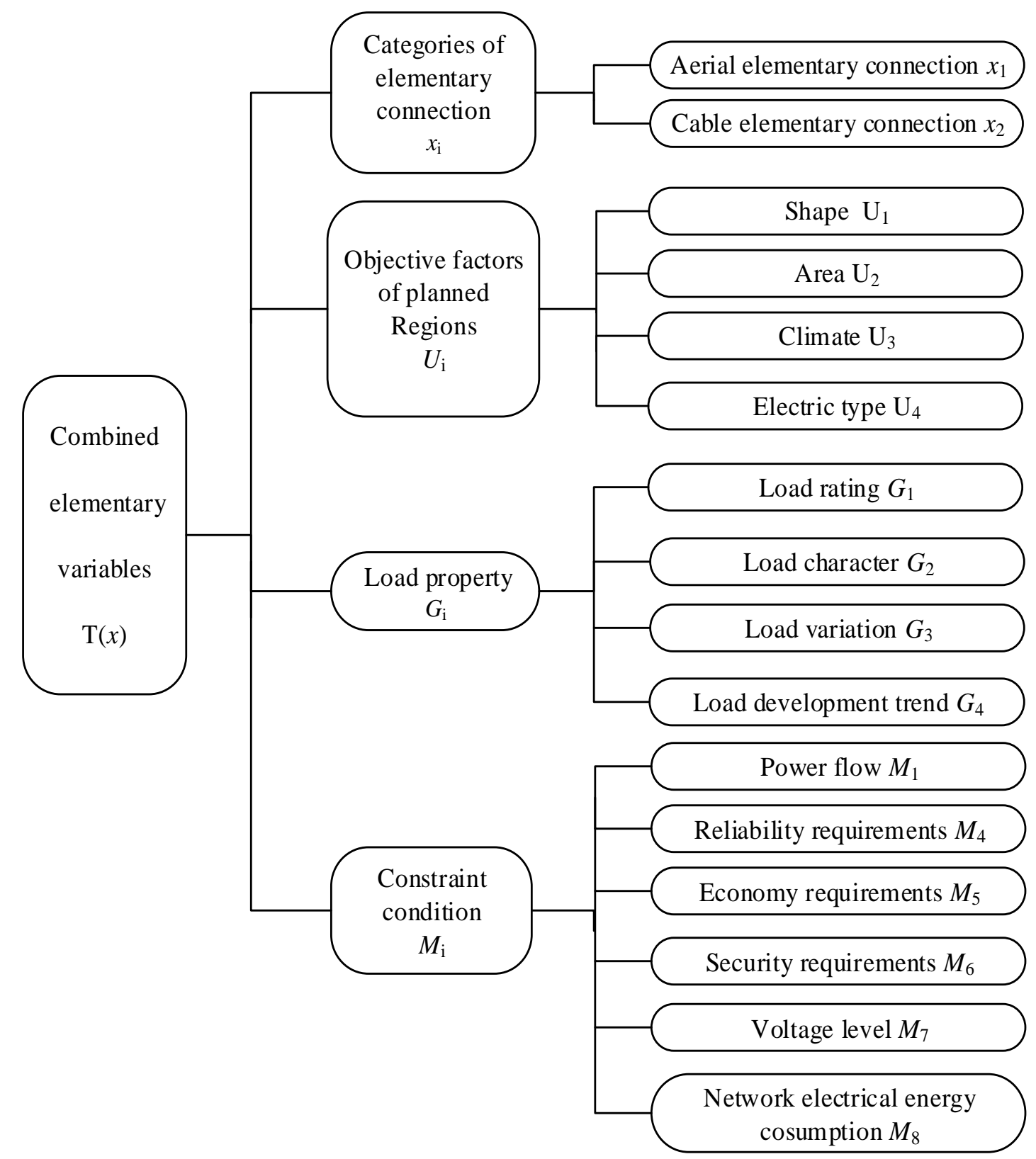

Figure 3. Combined variables of elementary connection

Steps of Build Connection Method of Distribution Network by Elementary Connection. 1) Forecast a kind of electricity area for load, based on annual electricity consumption electricity area, by investigating each load types and each type the number of users to calculate the predictive load value.

2) According to the load forecasting, determine the level of substation capacity, the number of new substation, the substation location and planning, making its meet the requirements of the load level and already some technical constraints, and making the optimal economic indicators.

3) According to the source of capacity, load rate, line number from substation site selection constant volume and the load capacity of transformer substation from load forecasting. Using the elementary connection method determine how many primitive wirings from power to the load for power supply can meet the requirements. Through the formula 11, we can calculate the number of elementary connection.

$$
N=\frac{P_{2}}{P_{1} \theta / n}
$$


$\mathrm{P}_{2}$.

Where Source capacity noted $\mathrm{P}_{1}$, load factor noted $\theta$ and the number of wires on substation noted

4) According to the number of primitive connection, determining its primitive connection type and planning the needs of geographical conditions, analysing the distribution network wiring inherent in the various constraint conditions, screening to get the typical connection mode satisfied conditions.

5) According to the above steps for distribution network connection method, we use FAHP to evaluate and get the optional connection method from safety, reliability and economy aspect.

\section{Conclusion}

According to elementary connection thought in other areas of the proposed primitive wiring thinking, planning area suitable for the formation of a load demand distribution network wiring, thus avoiding the inherent shortcomings of each existing wiring, breaking the traditional distribution planning network. Elementary connection are the basic wiring of power supply, with units, integrity and combinatorial. Different combinations of primitives can be defined as a primitive collection, which also meet the basic operations and basic nature of the classic collection.

\section{References}

[1] Wang wei, Zhang li-zi \& Ma xiu fan, (2006) "Based on the theory of structural units of medium voltage distribution network connection mode". Automation of electric power system, Vol. 30, No.11, pp35-39.

[2] Liu Xiang jun, MA Shuang \& Xu Gang, (2012) "Formation of Typical Connection Mode for Distribution Network by Elementary Connection Model”, Power System Technology, Vol.36, No. 2, pp59-63.

[3] Si J D, Tang E T, Xu G. Safety research in typical connection models of distribution system[C]//Asia-Pacific Power and Energy Engineering Conference. Chengdu, China IEEE Power \& Energy Society (PES), pp1-4.

[4] Zhang xuemei, Guo zhizhong, "Distribution network security analysis of k $(n-1+1)$ criteria", relay journal, Vol.15, No.2, pp9-12.

[5] Liu Wei, Guo zhizhong, "Distribution network security research", Proceedings of the CSEE, Vol.23, No.8, pp85-90.

[6] Liu zifa, Guo huimeng, Li mengyu, "Different wiring mode power grid blackout risk research", Electric power construction, Vol.35, No.8, pp25-31.

[7] Ma xiufan, Wang wei, Zhang lizi, "Urban distribution network connection mode research", Journal Of Northeast China Institute Of Electric Power Engineering, Vol.25, No.2, pp33-36

[8] Chen zhongyang, Wei qiong, Guo jiayi, "Primitive build connection mode of distribution network reliability analysis", East China electric power, Vol.42, No.9, pp1751-1753

[9] Nie hongzhan, Lyu pan, "Transmission network planning based on artificial fish algorithm", Advanced Technology of Electrical Engineering and Energy, Vol.27, No.2, pp11-15

[10] Pan feng, Zhang yujun, Zhou min, "A comprehensive evaluation hierarchy for MV distribution network based on connection modes", Power System Protection and Control, Vol.37 No.19, pp19-22

[11] Ba chao, "The smart grid power flow calculation and optimization", North-eastern university.

[12] Wang chengshan, Wang saiyi, "Different medium voltage distribution network connection mode of economy and reliability analysis", Automation of Electric Power Systems, Vol. 26, No.24, pp34-39 
[13] Chen changsong, "Micro net energy management model based on improved genetic algorithm", TRANSACTIONS OF CHINA ELECTROTECHNICAL SOCIETY, Vol.28, No. 4,pp196-201 